\title{
An Engagement Strategy for Teaching Computing Concepts
}

\author{
El Sayed Mahmoud \\ Sheridan College Institute of Technology and Advanced Learning \\ Canada
}

\begin{abstract}
The research work in this paper investigates a new teaching strategy that uses active learning through play to increase students' uptake of learning computing concepts. The strategy promotes student engagement through playing a customized Jenga game. The game consists of a set of blocks, one side of each block is covered with a piece of dry-erase tape to allow erasing and writing on the blocks. This allows instructors to reuse this editable Jenga for developing their own game-based learning activities. The editable Jenga can be used without writining if needed. Three sample activities with writing have been developed and conducted to test the strategy experimentally in addition to a fourth activity without writing any terms on the Jenga blocks. The test results showed that the strategy improves the class average and promotes the student engagement. A survey has been conducted to get students' feedback on the strategy. The survey results demonstrated that students like the play-based strategy. The contribution of this paper is the development of a teaching strategy that improves students' engagement which in turn helps students to learn important computing concepts.
\end{abstract}

\section{Introduction}

Games can improve students' uptake of learning in ways that regular lectures cannot [1,2, and 3]. Play is voluntary and enjoyable [3] which in turn facilitates and promotes student engagement [4]. Game-based learning enables students to practice on working in process-based environments because games are process-oriented and rule-governed. However, the positive effects of using games on learning cannot be generalized to all areas or games [5]. Over the last few years, research showed that using games in classrooms improves student engagement for specific subjects such as Math and English [6, 7, and 8]. The most important game's feature is that play promotes student's creativity. Each student should construct his own method that enables him to win. This method could be refined and mapped to a particular learning outcome space by group discussion. These gaming features motivate me to propose a play-based teaching strategy for teaching computing concepts at the School of Applied Computing.
This project aims to investigate a play-based teaching strategy that promotes learning through play at the School of Applied Computing. The strategy is to use editable Jenga blocks and other necessary tools that enable constructing customized versions of Jenga game for learning computing concepts and acquiring relevant skills. The deliverables of the project include this paper and a Jenga toolbox that contains editable Jenga game sets, dry-erase markers and examples of slightly modified versions of Jenga games for promoting learning through play. The toolbox enables professors to design various activities that help students to learn different concepts through play.

Jenga is a simple game that promotes the development of physical and mental skills. The game requires players to take turns removing one block at a time from a tower constructed of fifty four blocks. Each block that is removed is then balanced on top of the tower. The game was selected for this project because it teaches students a lot about solving multiobjective problems that can be mapped to various design problems in applied computing through analogy. The game was created by Leslie Scott, and currently is marketed by Hasbro.

\section{The strategy}

The strategy is to involve students in a customized Jenga game competition that constructs an active learning environment. This environment promotes student engagement and enables collaborative learning. Instructor prepares several Jenga game sets for the competition. He writes various sentences on a particular topic to the Jenga block sets. This allows students to read the sentences one by one randomly. The sentences written on each game set should cover a specific concept relevant to the topic. The sentences will be written to sticky labels too. A blank concept map should be prepared. Students use the labels to fill out the concept map.

\subsection{The strategy guidelines}

The class is split into groups so that each group uses a different Jenga game set that focuses on a particular concept. Each group removes one block a time from their tower, searches for a label that contains the sentence seen on the block, and sticks 
the label on the appropriate position on the blank concept map then stacks the block at the top of the tower to create a new layer. Each group uses the sentences to construct a concept map/model on the concepts targeted by their game set. Each group changes their game set every seven minutes by exchanging their place with the closest group located on their left. This rotation enables each group to explore each game set and learn from the concept maps created by the other groups. Students are not allowed to stack the removed block before adding the label to the map. The winner group should stick the maximum number of labels and reach the highest height in all games. We can allow students to write the sentences to the concept map directly instead of sticking labels. All students should join a gallery walk to explore the concept maps of the other groups and prepare for a group discussion. Jenga blocks could be with writing in some activities like activity 4 presented in this paper.

\section{The strategy tactics}

The strategy tactics are to (1) identify an editing technique that enable instructors to write text on the blocks; (2) design sample activities including abstract maps that could be used for teaching various topics. These activities could be used as examples that inspire applied computing instructors to develop their own activities and (3) Test the strategy.

\subsection{The strategy tactics}

My initial idea for creating editable Jenga was to paint the Jenga blocks using the dry-eraser paint see Figure 1. However, upon further investigation I ran into two issues. Firstly, there are left over smudges of ink on the blocks after erasing the text. Secondly there is not a lot of writing space on the regular wooden Jenga blocks. The large wooden Jenga cannot be manipulated for safety and mobility reasons. The weight of the large wooden Jenga could reach $20 \mathrm{lb}$ which is not safe when the tower falls.

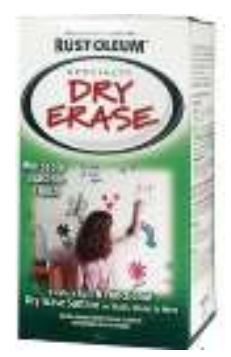

Figure 1. Dry erase paint

To address these problems, I have used dry-erase tape and found it easier than using the paint as shown in Figure 2. This tape could adhere to wood, metal and cardboard. This inspired me to use Xlarge Jenga which is made from cardboard. The Xlarge Jenga provided enough writing space for complex sentences. Moreover, it has a light weight $(1.5 \mathrm{~kg})$ which improves the mobility of the game and keeps it safe when the tower falls.

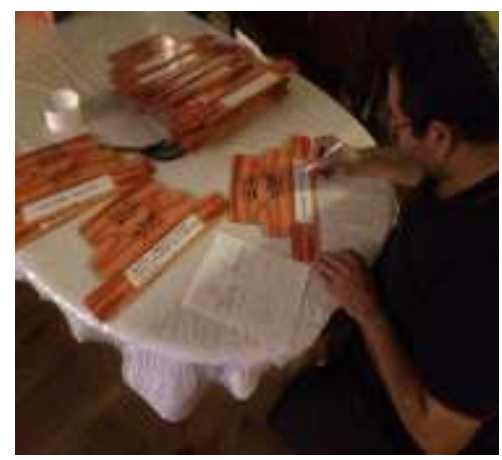

Figure 2. Preparing editable Jenga

I was browsing Toys R us and saw Jenga Quake which is made from plastic and contains a motor that randomly triggers a vibration causing the blocks to move slightly, see Figure 3. I have included this type of Jenga in the game-based learning toolbox for future activities that could help students to learn more about robust software development.

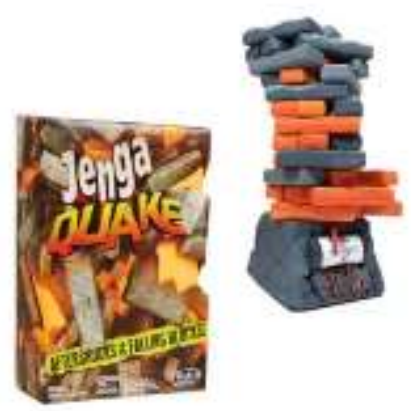

Figure 3. Xlarge Jenga (made from plastic)

\subsection{Sample activities}

Four sample activities have been designed to help students to learn through playing Jenga. The activities are designed to support learning abstract topics such as identifying relationships among objects and comparing several objects. This abstraction facilitates customizing the activities for various subjects and disciplines in future. The first three activities have been conducted in summer 2016 to engage three-year diploma students to object oriented system analysis concepts. The fourth activity conducted on winter 2017 to engage computer degree students to data structure and algorithms concepts. The session plan and the four activities are presented in the following sections. 
The first activity added to the toolbox is titled describe state chart and referred with $\mathrm{J} 1$. The activity description is shown in Figure 4. The activity helps students to establish concept map for the concepts and objects written on the picked Jenga block while they are playing the game.

\section{Activity 1: Describe state chart (J1)}

The purpose of playing $\mathrm{J} 1$ is to construct a concept map that describe state chart properly. The blocks of this Jenga game set reading many terms related to state chart concept. Each student in your group is required to do the following in his turn:

- Pull a block

- Read the text located on the block

- Insert this text into an oval on the provided sheet

- Connect the new oval to the previous ovals (if it is not the first).

- The arrows that connect the ovals must be labelled using one of the following verbs/phrases: models, form, are represented by, are, are when an object, lasts for, change by, could include, must have, change

- Add the block to the top of the tower

Playing and scoring rules:

- The tower must not fall and if a student touches a block he must play it

- The game ends when the tower falls or after the 7 minutes are complete

- A score will be assigned to each concept map to determine the winner team ( 5 pts per correct item).

Figure 4. An activity that helps students to identify relationships among objects

\section{Activity 2: Identify differences among Software Objects (J2)}

The purpose of playing $\mathrm{J} 2$ is to construct a table that compares a state chart and a sequence diagram. The blocks of this Jenga game set reading many features related to the two diagrams. Each student in your group is required to do the following in his turn:

- Pull a block

- Read the text located on the block

- Write this text to the appropriate side on the table (state chart or sequence)

- Add the block at the top of the tower

Playing and scoring rules:

- The tower should not fall and if a student touches a block he must play it

- The game ends when the tower fall or after 7 minutes

- A score will be assigned to each concept map to determine the winner team (5pts per correct item).

Figure 5. An activity that helps students identify differences between two objects
The second activity added to the toolbox is titled identifying differences among software objects and referred with $\mathbf{J} 2$. The activity is described in Figure 5. The activity helps students to establish a comparison table that identify and compare the features of software objects while they are playing the game $\mathbf{J} 2$.

The third activity added to the toolbox is titled identify relationships among objects. The activity description is shown in Figure 6. The activity helps students to figure out a missing object from a given concept map while they are playing the game J3. Students continue to play the Jenga game and read the object names written on the Jenga blocks. If they found an object fit the place of a missing one in the given concept map, they should insert it into the appropriate place and identify the relationship of this object and the surrounding objects. See the detailed instructions of the activity on Figure 6.

\section{Activity3: identify relationships among objects (J3)}

The purpose of playing $\mathrm{J} 3$ is to identify the relationships among requirement models and design models. A diagram with empty rectangles is provided and each group is required to fill the empty rectangles by the appropriate a model. The blocks of this Jenga game reading all requirement and design models. Each student in your group is required to do the following in his turn:

- Pull a block

- $\quad$ Read the model name on the block

- write model name to the appropriate rectangle

- add the block at the top of the tower

\section{Playing and scoring rules:}

- The tower should not fall and if a student touch a block he must play it

- The game ends when the tower fall or after 7 minutes

- A score will be assigned to each concept map to determine the winner team (5pts per correct item).

Figure 6. Activity 3 that helps students to learn how to identify relationships among objects

The three activities have been conducted during a single session. the session plan was prepared and handed to the students. The plan clarifies the purpose of the session and determines the expected time to complete the session. The session plan is shown in Figure 7.

The fourth activity has been designed to engage computer science students to the data structure and algorithm concepts. The idea is to use Jenga game as a metaphor for a multi-objective optimization problem. Students are expected to import the required skills for handling trade-offs from the game. The activity description is shown in Figure 8. 


\subsection{Test the strategy}

The three activities (see Figures 4, 5, and 6) were conducted by the students of the course titled: Object Oriented Methodologies (SYST39409) in summer 2016 at Davis campus of Sheridan. Quiz 3 was designed to test students on state charts diagram including relevant diagrams which is the topic targeted by the activities.

\section{Session plan for the first three activities}

The purpose of the session

This session aims to help students achieve the following learning objectives:

(1) To describe a state chart

(2) To identify the difference between a sequence diagram and a state chart

(3) To identify the relationships among requirement models and design models

Through the play of Jenga.

Expected Time: 45 minutes

\section{Activity Description}

1. Three Jenga game sets $\mathrm{J} 1, \mathrm{~J} 2$, and $\mathrm{J} 3$ will be used during this session. The blocks of each game set read sentences that are relevant to one of the target learning objectives listed above

2. The class will be split into 3 groups G1, G2, and G3

3. Each group will play a game for 7 minutes and then rotate to play the other games. For example: G1 plays $\mathrm{J} 1, \mathrm{G} 2$ plays $\mathrm{G} 2$, and $\mathrm{G} 3$ plays $\mathrm{J} 3$. After 7 minutes $\mathrm{G}$ 1 plays $\mathrm{J} 2, \mathrm{G} 2$ plays $\mathrm{J} 3$, and G3 plays J1. After another 7 minutes G1 plays J3, G2 plays J1, and G1 plays J2. (21 Minutes in total)

4. Each group will develop a concept map using the sentences written on the blocks of $\mathrm{J} 1$, develop a comparison table using the sentences from blocks of $\mathrm{J} 2$, and insert the diagram names written on the blocks of $\mathrm{J} 3$ into the appropriate position on the chart associated with $\mathrm{J} 3$.

How to play the Game while write the information found on each block to the sheet

1. Each time a student pulls the block they need to read a sentence

2. Write the sentence to the appropriate place on the sheet

3. Add the block at the top of the tower

4. Repeat steps from 1 to 3 till the 7 minutes end.

5. Each group posts their sheet

6. Groups will rotate to exchange games

\section{Gallery Walk and Discussion}

All students participate in a Gallery Walk in which they will read other groups' work and identify the following: (9 min)

- A common idea

- A unique idea

- An interesting idea

- A new piece of information

\section{Discussion:}

All students are welcome to share information they gathered from the activity ( $15 \mathrm{~min}$ )

Figure 7. session plan sample for running the first three activities together
To evaluate the strategy impact on student learning' uptake, the quiz 3 marks of the summer 2016 group are compared to the marks of a control group of the same course held in fall 2015. The two groups used exactly the same quiz and have been taught by myself at the same campus. The only difference between the two groups is that the Jenga learning activity was conducted by the summer 2016 group while no game-based activities were used in fall 2015. Additionally, a survey has been conducted to investigate the student feedback on the strategy.

\section{Activity 4: multi-objective optimization}

The purpose of this activity to identify the relationship between the Algorithm and data structure in the light of the trade-off between multiple objectives.

Expected time:40 minutes

Step 1: Watch the vedio at

https://www.youtube.com/watch?v=4w5kvC-zngY

Step 2: answer the question: what are the conflicting objectives in this game?

Step 3: play it (2 groups will compete for 3 minutes then each group will answer the following questions):

- What are the challenges?

- Does each move changes the structure of the tower?

- Is it important to consider the impact of previous movements when proposing a new move?

- Which is the best method for better results

- $\quad$ search for a complete set of movements that guarantee to keep balance and maximize the height, or

- Search for a new move after completing each move.

Step 4: what if we want build a closet instead of the tower so we are looking for empty spaces not the height. Each team should try to do so

Step 5: Each group will be given a ruler as an additional building unit.

Step 6: Each team should answer the questions: what is the impact of the new building unit? Does it fit the new goal (building a closet?)

Figure 8. A Jenga-based activity for learning how to solve multi-objective problems

The fourth activity (see Figure 8) was conducted by the students of the course titled: data structures and algorithms (PROG23672) in winter 2017 at Oakville campus of Sheridan. The engagement of the students during this activity was observed.

\section{Results and analysis}

This section shows and analyzes the results of the following: (1) a quiz on the topics taught using the strategy compared to the results of the same quiz 
without using the strategy; (2) a survey that determines how much students like the strategy, and (3) the observations of the author during conducting the activity. The results are discussed in the following sections

\subsection{The strategy improves the class average}

The average marks of the summer 2016 group are greater than the corresponding average marks of the fall 2015 group, see figure 9. This improvement could be attributed to the student engagement with the Jenga game activity (the strategy). Students read the statechart-related sentences from the Jenga blocks then insert them into the appropriate places on the concept map prepared for the statechartshould be indented.

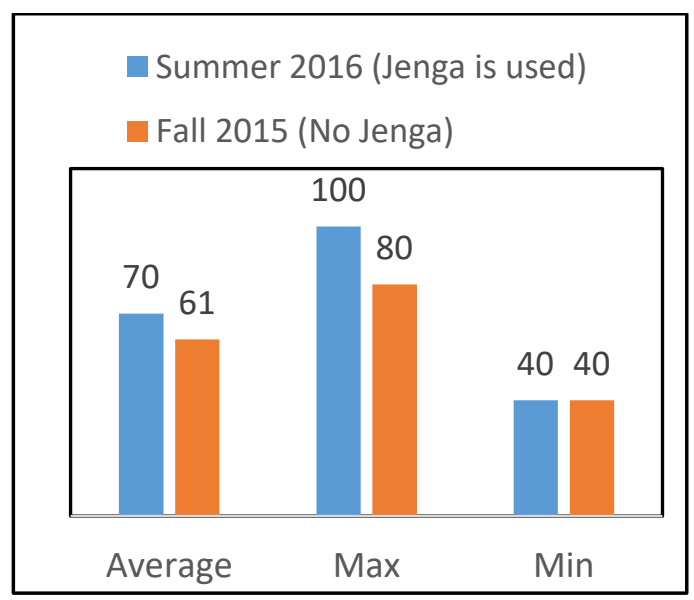

Figure 9. Marks of Quiz3 of two SYST39409 groups: summer2016 and fall 2015

In this activity, we ask questions that allow students' sense of purpose to evolve from their collaborative play. The open-ended discussion conducted after the game seems to inspire students to seek out and create answers for themselves based on their experience. This game allows students to build living models using their own experiences (languages) for the discussed concepts. These models are assets for students' uptake of learning. The value of these models increases when the student continues to care about updating these models to represent his recent knowledge

\subsection{The strategy promotes student engagement}

The student engagement to the activity was observed. Students were seriously focus on playing the game and building the concept maps. The engagement was shown by many student actions such as: a student picks up a block while the other two students have prepared to catch the tower if it happens to fall. Another example noticed is that students who are amused by the unstable tower (see Figures 10 and 11).

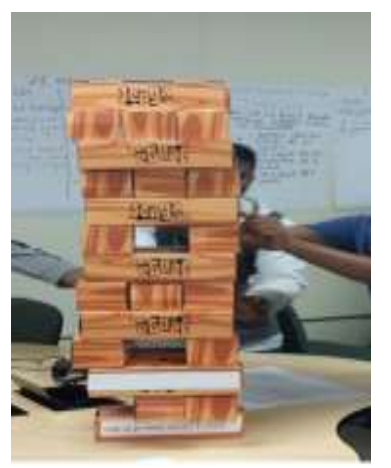

Figure 10. Students engaged to the game

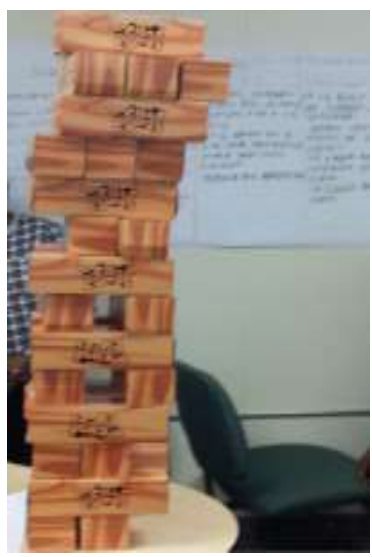

Figure 11. Unstable tower

Figure 12 shows the tower when it has fallen. The tower falling has taught students about the significance of each move they made, meaning one bad move could cause the entire tower to collapse. Students realised that they must care about every move which is very similar to the principles of designing an algorithm.

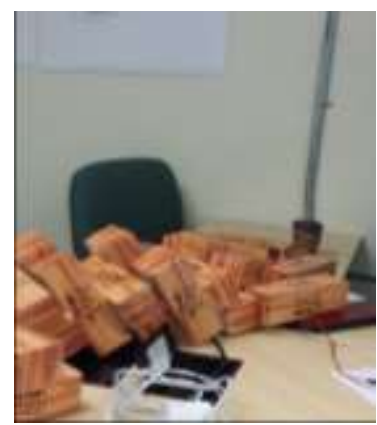

Figure 12. Fallen tower - a failure experience 


\subsection{The strategy promotes creativity}

The strategy promotes the creativity because it provides opportunities to create new ideas.

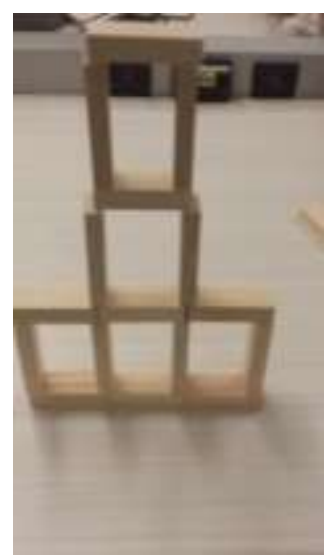

Figure 13. A new structure constructed using Jenga blocks

Figure 13 shows a new structure using the same Jenga blocks was produced by the students to meet different requirements for the fourth activity. This activity used the regular size of Jenga and focused on mapping the student skills from the game to the data structure design context. Figure 14 shows the concept map resulted from activity $\mathrm{J} 1$. This concept map contains relationships that were not discussed in the class or presented in the textbook. Students select the block randomly which in turn provides students with a random list of concepts and they think deeply to identify the relationships among these list of concepts.

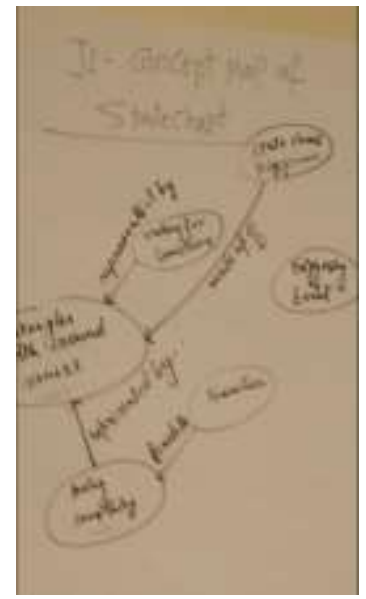

Figure 14. Concept map
The ultimate goal is to promote diversity in learning activities to accommodate different learning styles of students. A survey was designed to investigate student feedback on the Jenga activity and the adopted teaching approach generally. Figure 15 shows the survey questions where 1 indicates extremely well and 5 indicates not well at all.

Note: This activity is part of an education research project and your work may be used anonymously.

\section{Question 1}

How well does the activity offered today help you to learn the proposed concepts?

$\begin{array}{lllll}1 & 2 & 3 & 4 & 5\end{array}$

\section{Question 2}

How well does the activity match your learning style?

$\begin{array}{lllll}1 & 2 & 3 & 4 & 5\end{array}$

Question 3

In the second part of this course (from week 8 till now), how well does the overall approach to learning work for you?

$\begin{array}{lllll}1 & 2 & 3 & 4 & 5\end{array}$

Question 4

How comfortable are you in asking for help from the instructor?

$\begin{array}{lllll}1 & 2 & 3 & 4 & 5\end{array}$

Question 5

How well this activity does helped you to develop critical thinking skills?

$\begin{array}{lrrrr}1 & 2 & 3 & 4 & 5 \\ \text { Question } 6 & & \\ \text { Which Jenga game set gives more fun and help in } \\ \text { learning? } \\ 1\end{array}$

Figure 15. survey about the strategy activities

The survey results showed that students like the Jenga activity which helped them to learn more than 
the regular lecture. This result has been demonstrated by the analysis of students' answers for the first five questions of the survey. Fourteen students out of twenty-one stated that the Jenga activity fits their learning style extremely well (see Figure 16).

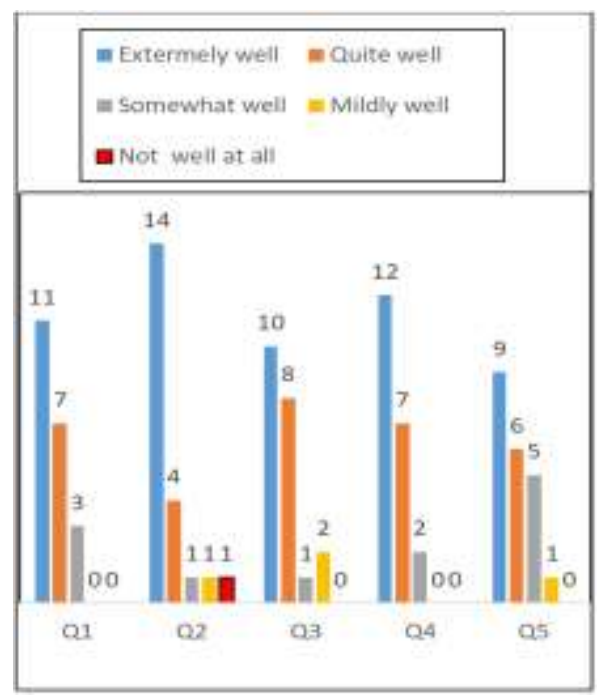

Figure 16. The students' answers for the first five questions of the survey

The total number of students who selected extremely well and quite well reflects how well the activity alleviates different students to learn. Figure 17 shows the survey results after merging these two student groups (extremely well and quite well). For the first three questions, the total number is eighteen students out of twenty-one students. For questions four and five, the total numbers are nineteen and fifteen respectively. This confirms that the majority of the students liked the activity and learned from it.

\subsection{No significant difference between regular size and the $\mathrm{XL}$ size of Jenga in terms of engagement}

The answers of question 6 show that students enjoyed the two Jenga sizes. Eleven students preferred XL size and ten students preferred the regular size. Figures 18 and 19 show the student engagement with the wooden Jenga too. In Figure 17 students is very cautious while picking up the block. In Figure 19, two students point to the different blocks to be selected. This indicates that the size of the Jenga does not have a significant impact on the student engagement.

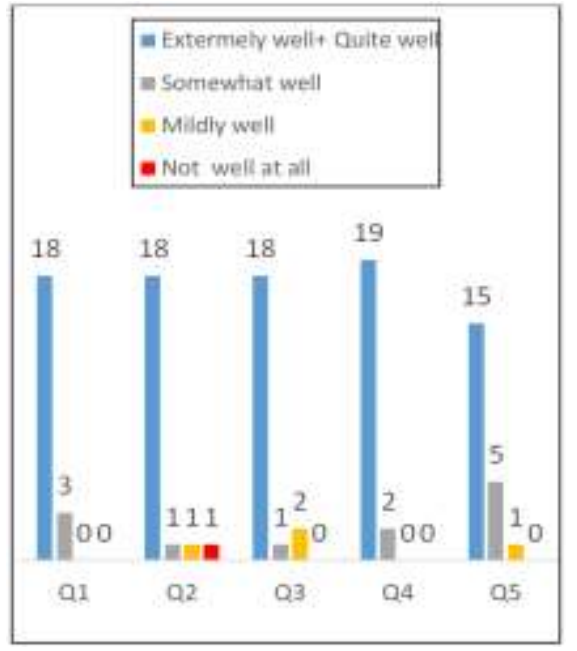

Figure 17. The student answers for the first five questions -combining the extremely well and quite well options

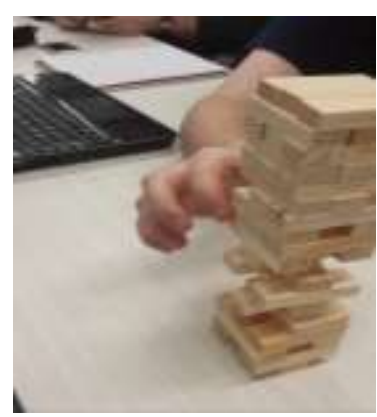

Figure 18. a student picks a block

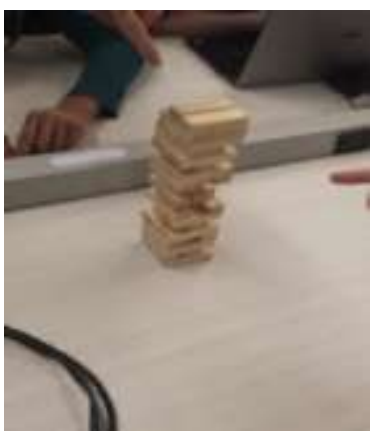

Figure 19. two students suggest a block to be removed

\section{Deliverables}

The deliverables of this project include: (1) this report; (2) two editable cardboard XL Jenga; (3) one editable wooden regular size Jenga; (4) dry-erase markers; (5) board eraser; (6) white-board spray cleaner; (7) instructions of three sample activities 
and (8) a box for keeping all components. Figure 20 shows these components where each component is referenced by its order in this list.

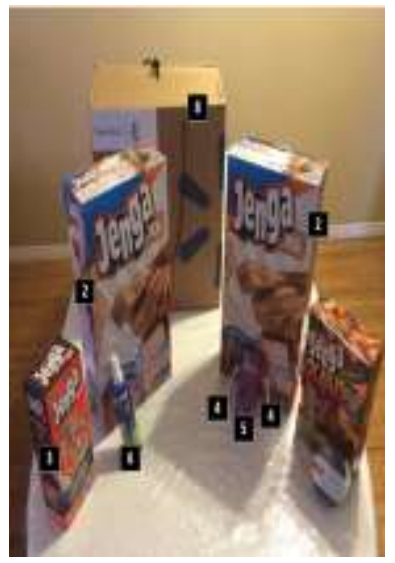

Figure 20. Contents of the Jenga Applied computing (JAC) Toolbox

\section{Conclusions}

A new strategy for teaching computer science topics was investigated. The strategy aims to promote engagement through playing a customized Jenga game. The customized Jenga constructs an active learning environment that reveals a model for concepts to learn. This allows students to perceive the model in different ways that fit their diverse experiences. Dry-erase tape, fine dry-erase markers, and cardboard XL .Jenga were used to create an editable, large and light weight version of the Jenga. Three activities were designed and conducted in the course SYST39409 held in summer 2016 to test the strategy experimentally. The marks of a quiz on the topics targeted by the activities were used to evaluate the strategy impact on the students' uptake of learning. These marks were compared to the marks of a control group which studied the same material in fall 2015 but without applying the strategy. The comparison demonstrated an improvement in the class average. Additionally, a survey was conducted to get students feedback on the strategy. The survey showed that students like the strategy because they can learn from it more than traditional lectures. The survey also showed that students like the regular and $\mathrm{XL}$ sizes of Jenga.

\section{Future work}

This work developed a framework of tools and instructions that provide the basis for an effective development environment where robust game-based learning activities can be developed to facilitate learning complex concepts. To get closer to this environment this work requires further investigation in two directions: design more activities and use a $3 \mathrm{D}$ printer that enables printing customized blocks.

\section{References}

[1] Connolly, Thomas M., et al. "A systematic literature review of empirical evidence on computer games and serious games." Computers \& Education 59.2 (2012): 661686.

[2] Kapp, Karl. "Once Again, Games Can and Do Teach!." Learning Solutions Magazine (2013).

[3] Krishan, Yuyutsu, and Shen Zhiqi. "An Effective Approach to Educational Games." Proceedings of the URECA@ NTU (2009): 10.

[4] Wouters, Pieter, et al. "A meta-analysis of the cognitive and motivational effects of serious games." Journal of Educational Psychology 105.2 (2013): 249.

[5] Hays, Robert T. The effectiveness of instructional games: A literature review and discussion. No. NAWCTSD-TR-2005-004. NAVAL AIR WARFARE CENTER TRAINING SYSTEMS DIV ORLANDO FL, 2005.

[6] Honeyford, Michelle A., and Karen Boyd. "Learning Through Play." Journal of Adolescent \& Adult Literacy 59.1 (2015): 63-73.

[7] Enyedy, Noel, et al. "Learning physics through play in an augmented reality environment." International Journal of Computer-Supported Collaborative Learning 7.3 (2012): 347-378.

[8] Sharma, Mahesh. "Prerequisite Skills and Mathematics Learning: Role of Games in Learning Mathematics." http://interlakes.org/images/stories/documents/iles/List_of _Games_2013R_1.pdf, (Access Date: 07 Feb, 2017). 